\title{
Click Chemistry in Carbon Nanoreactors
}

\author{
Graham A. Rance,* William A. Solomonsz, Andrei N. Khlobystov* \\ Received (in $X X X, X X X)$ Xth $X X X X X X X X X 20 X X$, Accepted Xth $X X X X X X X X X 20 X X$ \\ DOI: 10.1039/b000000x
}

${ }_{5}$ Copper-nanoparticle catalytic centres anchored at the graphitic step-edges within hollow carbon nanoreactors exhibit superior activity and stability in cycloaddition reactions as compared to catalytic centres outside the nanoreactors. Nanoscale confinement enables efficient ${ }_{10}$ recycling of the catalyst in preparative-scale synthesis without significant changes in activity.

Confinement of molecules by restricting the available spatial volume down to the nanoscale provides a powerful methodology to control their physical properties and chemical reactivity. ${ }^{[1-4]}$

${ }_{15}$ Over the past three decades, a variety of molecular nanocontainers, including cyclodextrins, cavitands, calixarenes, cucurbiturils and supramolecular/coordination cages, ${ }^{[5]}$ have been developed to replicate enzymatic-type nanoscale confinement, which drastically alters concentration, pressure and alignment of 20 reactants and lowers the activation barriers of chemical reactions as compared to the bulk phase (solution or gas). ${ }^{[1]}$

Typically, molecular containers are synthetically tailored for a particular class of guest-molecules and consequently require intricate design and lengthy preparation. Furthermore, their

25 thermal and chemical stabilities limit their applications to a narrow range of conditions, due to the chemically reactive functional groups of the host container (e.g. $-\mathrm{OH},-\mathrm{NH}_{2}$ ). In contrast, hollow carbon nanostructures possess extremely high mechanical, chemical and thermal stability and the ability to

30 encapsulate the widest spectrum of guest-molecules due to ubiquitous van der Waals forces. The diameters of carbon nanotubes (CNT) can be readily selected to provide the optimum confinement for the reactants of a particular chemical reaction, which can be studied at either the macroscale ${ }^{[6-8]}$ or the 35 nanoscale. ${ }^{[9-11]}$ However, the use of CNT, whose internal diameters are typically in the range $1-10 \mathrm{~nm}$, in preparative scale reactions may result in substantial resistance to the transport of reactants and products to and from CNT, thus significantly thwarting kinetics of reactions ${ }^{[12,13]}$ and in some cases totally

40 precluding extraction of the products from within the internal cavity. ${ }^{[14]}$ Hollow graphitised carbon nanofibres (GNF) being significantly wider than CNT (internal diameters typically above $50 \mathrm{~nm}$ ) and always readily accessible for molecules, solve this problem of transport resistance. ${ }^{[15,16]}$ However, despite the fact 45 that the size of GNF significantly exceeds the typical dimensions of small organic molecules, which is expected to lead to the loss of confinement effects, it has been recently demonstrated that their unique internal structure consisting of a succession of 3-4

$\mathrm{nm}$ high steps formed by rolled-up sheets of graphene provide 50 effective "anchoring points" for molecules and nanoparticles ${ }^{[17]}$ and thus create localised nanoscale reaction environments, different to the bulk phase, while still allowing effective transport of molecules through the tubular structure of GNF. ${ }^{[15,16]}$

In this study, we demonstrate for the first time that 55 confinement in carbon nanoreactors has a remarkable stabilising effect on catalytic centres relative to catalysts deposited on the outer surfaces or dispersed in solution. Our novel nanoreactor catalysts can be readily recycled with retention of high catalytic activity, thus highlighting the importance of nanoreactors in ${ }_{60}$ preparative synthesis. Small dodecanethiolate-capped copper nanoparticles $(\mathrm{CuNP}, \mathrm{S} 1-\mathrm{S} 2 \dagger),{ }^{[18]}$ were chosen as catalysts and inserted into the inner channel of GNF using $n$-hexane and supercritical $\mathrm{CO}_{2}(\mathrm{~S} 3 \dagger)$. The resulting composite structure (designated as CuNP@GNF) contains the majority (> $90 \%$ ) of 65 the nanoparticles immobilised at the corrugated internal stepedges of the nanofibres as demonstrated by high resolution transmission electron microscopy (HR-TEM, Figures 1a-c and $\mathrm{S} 3 \dagger)$. In a control experiment, CuNP were deposited onto the exterior of GNF (CuNP/GNF). Systematic comparison enables 70 discrimination between the effects of support and confinement.
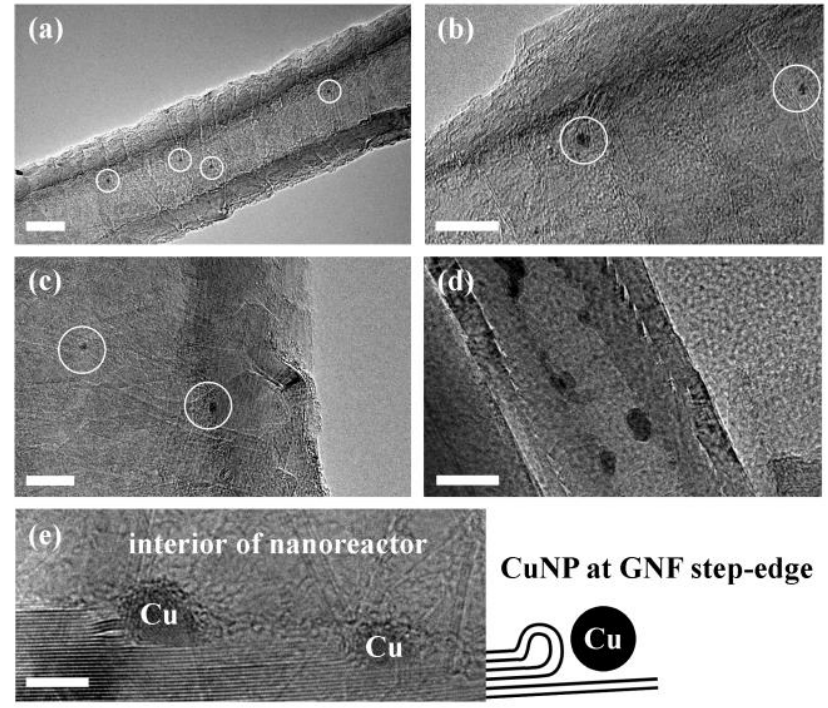

Figure 1. HR-TEM of CuNP@GNF. CuNP ( 3 nm) immobilised at the step-edges (a-c) increase in size $(\sim 20 \mathrm{~nm})$ following catalysis (d) or thermal treatment (e), but remain within the GNF inner channel. White circles guide the eye to the location of small ${ }_{75} \mathrm{CuNP}$. Scale bars are 20 (a,d), 10 (b,c) and $5 \mathrm{~nm}(\mathrm{e})$. 
The use of CuNP in catalysis is highly attractive due to the low cost of both precursors and the bulk metal (relative to the other noble metals) and the plethora of carbon-carbon and carbonheteroatom (oxygen, nitrogen, sulphur, selenium) bond-forming 5 reactions catalysed by nanoscale copper. ${ }^{[19]}$ For example, CuNP are very effective in the Huisgen cycloaddition of azides to alkynes $^{[20,21]}$ and was selected as a model reaction in our study to assess the use of GNF as nanoreactors (Table 1).

${ }_{10}$ Table 1. The Huisgen cycloaddition of azides to alkynes. ${ }^{\mathrm{a}}$

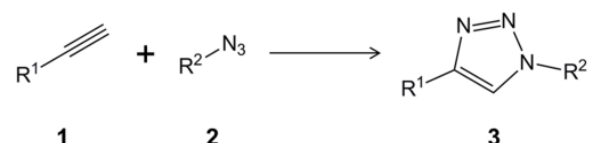

1

\begin{tabular}{|c|c|c|c|c|c|c|}
\hline & Catalyst & $\mathrm{R}^{1}$ & $\mathrm{R}^{2}$ & Product & $\mathrm{t} / \mathrm{hr}$ & Yield / \% \\
\hline 1 & GNF & $p-\mathrm{NO}_{2} \mathrm{Ph} \mathbf{1 a}$ & Bn 2a & $3 \mathbf{a}$ & 168 & 0 \\
\hline 2 & CuNP & 1a & $2 a$ & $3 \mathbf{a}$ & 48 & 96 \\
\hline 3 & $\mathrm{Cu}_{0.75} \mathrm{~A}_{\mathrm{g} 0.25} \mathrm{NP}$ & 1a & $2 a$ & 3a & 24 & 83 \\
\hline 4 & $\mathrm{Cu}_{0.50} \mathrm{Ag}_{0.50} \mathrm{NP}$ & $1 \mathrm{a}$ & $2 a$ & $3 \mathbf{a}$ & 24 & 99 \\
\hline 5 & $\mathrm{Cu}_{0.25} \mathrm{Ag}_{0.75} \mathrm{NP}$ & 1a & $2 a$ & $3 a$ & 24 & 99 \\
\hline 6 & $\mathrm{AgNP}$ & $1 \mathbf{a}$ & $2 \mathbf{a}$ & $3 \mathbf{a}$ & 168 & 0 \\
\hline 7 & $\mathrm{CuI}$ & 1a & $2 a$ & $3 \mathbf{a}$ & 24 & 95 \\
\hline 8 & $\mathrm{CuNP}^{\mathrm{b}}$ & 1a & $2 a$ & $3 a$ & 4 & 96 \\
\hline 9 & $\mathrm{CuNP}^{\mathrm{c}}$ & $1 \mathbf{a}$ & $2 \mathbf{a}$ & $3 a$ & 168 & 67 \\
\hline 10 & $\mathrm{CuNP}$ & $p-\mathrm{BrPh} \mathbf{1 b}$ & $2 a$ & $3 b$ & 48 & 94 \\
\hline 11 & $\mathrm{CuNP}$ & $\mathrm{Ph} \mathrm{1c}$ & $2 a$ & $3 c$ & 144 & 99 \\
\hline 12 & CuNP & $p$-MeOPh 1d & $2 \mathbf{a}$ & 3d & 144 & 95 \\
\hline 13 & CuNP & $p-\mathrm{NH}_{2} \mathrm{Ph} \mathbf{1 e}$ & $2 a$ & $3 e$ & 144 & 94 \\
\hline 14 & $\mathrm{CuNP}$ & Cy $\mathbf{1 f}$ & $2 a$ & 3f & 24 & 93 \\
\hline 15 & CuNP & $\mathrm{C}_{8} \mathrm{H}_{17} \mathbf{1 g}$ & $2 \mathbf{a}$ & $3 g$ & 66 & 97 \\
\hline 16 & CuNP & $\mathrm{OHCH}_{2} \mathbf{l h}$ & $2 \mathbf{a}$ & $3 h$ & 66 & 99 \\
\hline 17 & $\mathrm{CuNP}$ & $1 \mathrm{a}$ & $\mathrm{C}_{6} \mathrm{H}_{13} \mathbf{2 b}$ & $3 \mathbf{i}$ & 72 & 99 \\
\hline
\end{tabular}

a Standard conditions: alkyne $(0.1 \mathrm{mmol})$, azide $(0.1 \mathrm{mmol})$, catalyst $(2.5 \mathrm{~mol} \% \mathrm{Cu})$, triethylamine $(0.15 \mathrm{mmol}), \mathrm{C}_{6} \mathrm{D}_{6}(1.4$ $\mathrm{mL}), 40{ }^{\circ} \mathrm{C}(\mathrm{S} 4 \dagger) .{ }^{\mathrm{b}} 80{ }^{\circ} \mathrm{C} .{ }^{\mathrm{c}} \mathrm{MeOD}$.

${ }_{20}$ Our control experiments revealed that the addition of benzyl azide 2a to para-nitrophenylacetylene 1a proceeds only in the presence of copper, as we found no products of cycloaddition in empty GNF under our experimental conditions (entry 1, Table 1). In the presence of $\mathrm{CuNP}$ dispersed in solution the cycloaddition 25 afforded solely the 1,4-regioisomer of 1-benzyl-4-(4nitrophenyl)-1H-[1,2,3]-triazole $\mathbf{3 a}$, in near quantitative conversion (entry 2). The regioselectivity is unaffected by the source of copper, ${ }^{[2]}$ with a range of copper-silver alloy nanoparticles (entries 3-5 and S2 $\dagger$ ) and molecular salts (entry 7) 30 showing remarkable regioselectivity and activity similar to pure CuNP. Further experiments showed that the reaction can be performed under a range of different experimental conditions (entries 8-9) and is suitable for a wide variety of aromatic and aliphatic azides and alkynes (entries 10-17), clearly showing the

35 Hammett rule $(\mathrm{S} 5 \dagger)$. All of these measurements indicate that the CuNP-catalysed Huisgen cycloaddition is a stable, reproducible reaction suitable for the evaluation of nanoreactors and for studying the catalytic properties of confined catalysts. We, thus, monitored the addition of 2a to 1a using ${ }^{1} \mathrm{H}$ NMR spectroscopy 40 and compared the selectivity, activity and recyclability of CuNP,
CuNP/GNF and CuNP@GNF catalysts (Figure 2).
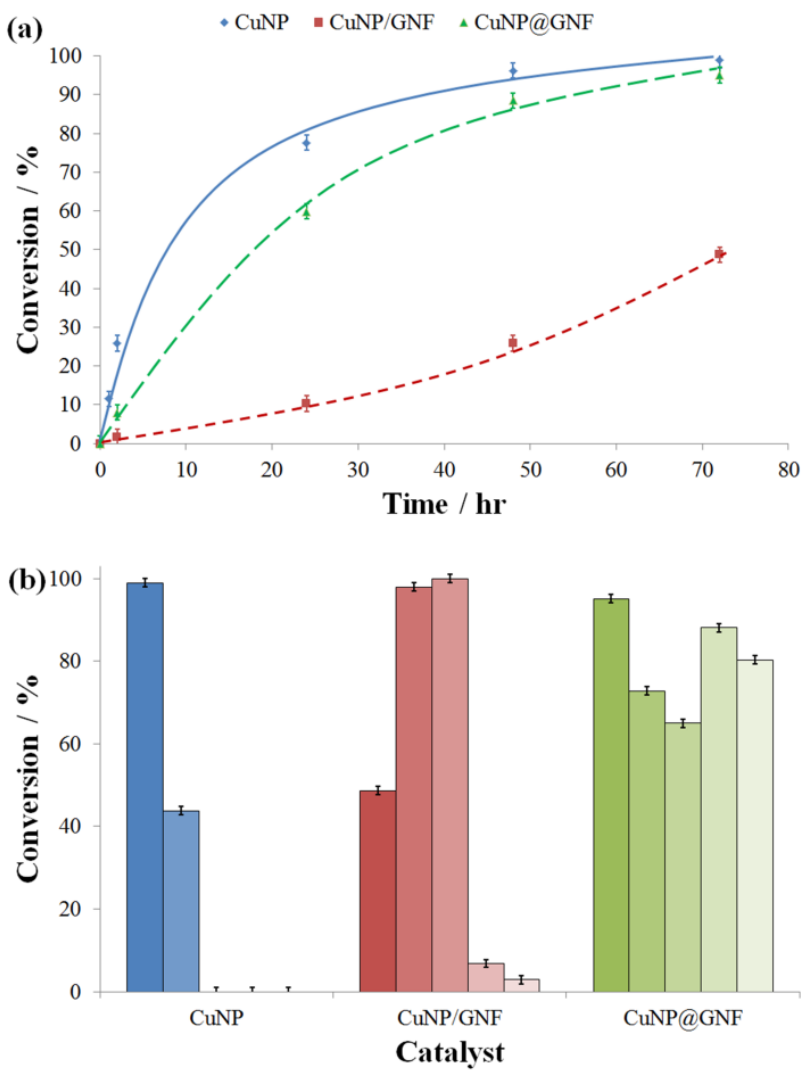

Figure 2. The comparative (a) kinetics and (b) recyclability over five cycles (measured as the conversion at $72 \mathrm{hr}$ ) for the copper catalysts dispersed in solution (CuNP), deposited on the surface ${ }_{45}(\mathrm{CuNP} / \mathrm{GNF})$ or inserted in nanoreactors $(\mathrm{CuNP} @ \mathrm{GNF})$.

Firstly, the sole 1,4-regioisomer was afforded under all experimental conditions investigated for nanoreactors. This represents the principal example of the cycloaddition reaction 50 conducted inside hollow carbon nanostructures and implies that the constrained steric environment imposed by the graphitic stepedge of GNF has no effect on the regioselectivity of the reaction. This contrasts with our previous observations for hydrosilylation reactions where confinement at the GNF step-edges resulted in 55 different reaction pathways. ${ }^{[16]}$

Secondly, the rate of formation of the 1,2,3-triazole product is significantly higher for CuNP@GNF (commensurate with CuNP) relative to $\mathrm{CuNP/GNF}$ (Figure 2a). This result is surprising as catalysts deposited on the outer surface of nanoreactors are more 60 accessible to reactant molecules than those embedded within nanoreactors. The increased reaction rate and improved conversion for CuNP@GNF can be attributed to an increased local concentration effect. Reactants $\mathbf{1}$ and $\mathbf{2}$ both possess aromatic groups enabling specific van der Waals interactions with ${ }_{65}$ the graphitic step-edges within GNF leading to heightened concentrations inside the nanoreactor cavity, relative to the bulk phase, and thus enhancement in kinetics of reactions. ${ }^{[15,16]}$

Thirdly and most significantly, the recyclability of CuNP@GNF nanoreactors relative to supported CuNP/GNF and 70 free-standing CuNP catalysts is much greater. Whilst the catalytic activity of CuNP and CuNP/GNF decreases rapidly after one and 
three cycles respectively, CuNP inside nanoreactors exhibit remarkably stable catalytic activity even after five cycles (Figure 2b). HR-TEM imaging of the CuNP@GNF structures after several catalytic cycles clearly illustrates that the initial small ${ }_{5} \mathrm{CuNP}$ undergo dynamic coalescence into much larger particles ( 20-30nm, Figure 1d and S6†), thus explaining the subtle variation in absolute activity across catalytic cycles, but critically remain anchored to the interior of GNF and therefore available for subsequent reactions (Scheme 1). In contrast, under the same 10 conditions CuNP are removed from the surfaces of nanofibres in $\mathrm{CuNP/GNF}$ ( $\mathrm{S} 6 \dagger$ ), due to dissolution of copper in the reaction mixture, thus leading to a continuous and eventual total loss of catalytic centres after only three cycles. In a series of control experiments, CuNP were separately grown to a larger size $(\sim 10-$ $1520 \mathrm{~nm}$, Figure 1e and S7†) inside and outside GNF prior to catalysis. These experiments confirmed that activity and recyclability of CuNP@GNF is not affected by the size of CuNP as long as they remain located inside nanoreactors $(\mathrm{S} 7 \dagger)$ and further emphasises the importance of confinement of the catalytic 20 centres within nanoreactors.

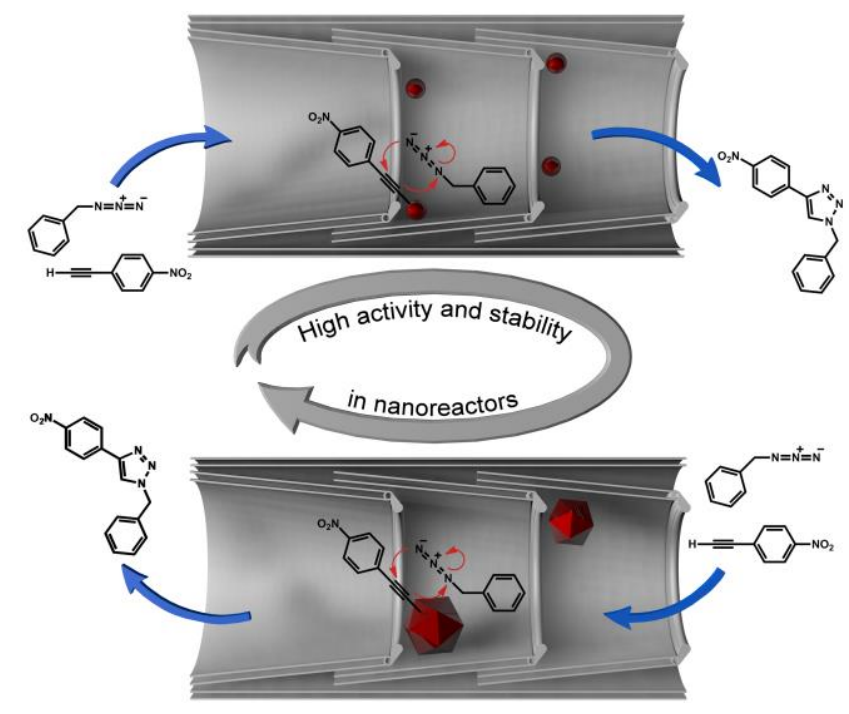

Scheme 1. The retention of high catalytic activity after multiple cycles using CuNP@GNF nanoreactor catalysts.

25 In conclusion, we have shown for the first time that carbon nanoreactors provide an excellent environment for cycloaddition reactions. The kinetics of reactions are accelerated and conversion rates improved in nanoreactors relative to catalysts deposited on the outer surfaces. Most significantly, catalytic 30 centres embedded in the nanoreactor cavity are stabilised by interactions with the nanoscale graphitic step-edges, which prevent the loss of catalyst during reactions, thus allowing efficient recycling of CuNP@GNF with retention of high catalytic activity cycle after cycle. This methodology can be 35 transferred to other types of reactions as the Brust-Schiffrin reductive synthesis of nanoparticles is applicable to a wide range of catalytically active transition metals, which can be readily inserted into GNF nanoreactors using the methodology described for CuNP in this study. Our findings broaden the spectrum of 40 preparative chemical transformations in carbon nanoreactors which present an ideal catalyst system for further exploration of the effects of nanoscale confinement on chemical processes, ensuring high selectivity, activity and recyclability of catalytic centres. Current development of nanotubes as nanoreactors is 45 very timely, as similar nanotube-nanoparticle composite structures have recently been demonstrated as magnetically controlled pipettes, ${ }^{[23]}$ liquid chromatographers ${ }^{[24]}$ and cellular endoscopes, ${ }^{[25]}$ which are fundamentally changing the way chemists study and make molecules.

50 The authors thank the ERC for supporting this research, the NNNC for access to TEM facilities and Mr Ahmed Alhadrami and Mr Scott Miners for technical assistance.

\section{Notes and references}

School of Chemistry, University of Nottingham, University Park, 55 Nottingham, UK. Fax: 44 1159513563; Tel: 44 1159513917; E-mail: graham.rance@nottingham.ac.uk, andrei.khlobystov@nottingham.ac.uk $\dagger$ Electronic Supplementary Information (ESI) available. See DOI: $10.1039 / \mathrm{b} 000000 \mathrm{x} /$

1 A.N. Khlobystov, ACS Nano 2011, 5, 9306-9312.

602 D.A. Britz, A.N. Khlobystov, Chem. Soc. Rev. 2006, 35, 637-659.

3 P. Serp, E. Castillejos, ChemCatChem 2010, 2, 41-47.

4 X. Pan, X. Bao, Acc. Chem. Rev. 2011, 44, 553-562.

5 U.D. Brinker, J.-L. Mieusset (Eds.), Molecular encapsulation: Organic reactions in constrained systems, 2010, Wiley, New York.

656 X. Pan, Z. Fan, W. Chen, Y. Ding, H. Luo, X. Bao, Nature Mater. 2007, 6, 507-511.

7 W. Chen, Z. Fan, X. Pan, X. Bao, J. Am. Chem. Soc. 2008, 130, 9414-9419.

8 E. Castillejos, P.-J. Debouttiere, L. Roiban, A. Solhy, V. Martinez, Y. 70 Kihn, O. Ersen, K. Philippot, B. Chaudret, P. Serp, Angew. Chem. 2009, 121, 2567-2571.

9 C.S. Allen, Y. Ito, A.W. Robertson, H. Shinohara, J.H. Warner, ACS Nano 2011, 5, 10084-10089.

10 A. Ilie, J.S. Bendall, K. Nagaoka, S. Egger, T. Nakayama, S. Crampin, ACS Nano 2011, 5, 2559-2569.

11 A. Ilie, S. Egger, S. Friedrichs, D.-J. ang, M.L.H. Green, Appl. Phys. Lett. 2007, 91, 253124.

12 S. Xu, W. Zhang, X. Liu, X. Han, X. Bao, J. Am. Chem. Soc. 2009, $131,13722-13727$.

8013 D.A. Newsome, D.S. Scholl, Nano Lett. 2006, 6, 2150-2153.

14 D.A. Britz, A.N. Khlobystov, K. Porfyrakis, A. Ardavan, G.A.D. Briggs, Chem. Commun. 2005, 37-39.

15 A. La Torre, M.C. Gimenez-Lopez, M. Fay, G.A. Rance, W.A. Solomonosz, T.W. Chamberlain, P.D. Brown, A.N. Khlobystov, ACS Nano 2012, 6, 2000-2007.

16 W.A. Solomonsz, G.A. Rance, M. Suyetin, A. La Torre, E. Bichoutskaia, A.N. Khlobystov, Chem. Eur. J. 2012, 18, 1318013187.

17 A. La Torre, M. Fay, G.A. Rance, M.C. Gimenez-Lopez, W.A. 90 Solomonsz, P.D. Brown, A.N. Khlobystov, Small 2012, 8, 1222 1228.

18 M. Brust, M. Walker, D. Bethell, D.J. Schiffrin, R. Whyman, J. Chem. Soc. Chem. Comm. 1994, 801-802.

19 B.C. Ranu, R. Dey, T. Chatterjee, S. Ahammed, Chemsuschem 2012 $95 \quad 5,22-44$

20 F. Alonso, Y. Moglie, G. Radivoy, M. Yus, Eur. J. Org. Chem. 2010, 1875-1884.

21 Z.F. Zhang, C.M. Dong, C.H. Yang, D. Hu, J. Long, L. Wang, H. Li, Y. Chen, D.L. Kong, Adv. Synth. Cat. 2010, 352, 1600-1604.

10022 V.V. Rostovtsev, L.G. Green, V.V. Fokin, K.B. Sharpless, Angew. Chem. Int. Ed. 2002, 41, 2596-2599.

23 J.R. Freedman, D. Mattia, G. Korneva, Y. Gogotsi, G. Friedman, A.K. Fontecchio, Appl. Phys. Lett. 2007, 90, 103108.

24 R. Singhal, V.M. Mochalin, M.R. Lukatskaya, G. Friedman, Y. Gogotsi, Sci. Rep. 2012, 2, 510.

25 J.J. Niu, M.G. Schrlau, G. Friedman, Y. Gogotsi, Small 2011, 7, 540545 . 